\title{
Uncertain-frequency detection: Cuing and condition of observation
}

\author{
DAVID M. JOHNSON and ERVIN R. HAFTER \\ University of California, Berkeley, California 94720
}

\begin{abstract}
Two experiments are described which support a two-factor model of the detection of signals of uncertain frequency. This model asserts that both the number of channels to be monitored (M) and the accuracy (or bandwidth, W) of that monitoring influence detection. The condition of observation, whether simultaneous or successive, was also varied within this paradigm. Stimuli consisted of tones presented with a background of filtered noise. A yes-no psychophysical procedure was employed. In Experiment 1, tonal cues were used to reduce uncertainty about $\mathrm{W}$ while holding $\mathrm{M}$ constant. This resulted in improved performance. In Experiment 2, the validity of the tonal cue was varied. Good cues improved performance, while bad cues harmed it. This latter finding suggested that reducing uncertainty about one possible signal increased uncertainty about the other.
\end{abstract}

An important feature of signal-detection theory is that uncertainty about features of a signal causes performance to decline. Evidence of the effect of uncertainty is seen in studies of auditory detection in which the signal, on any trial, may be one of a set of possible frequencies (Green, 1961; Green \& Swets, 1966; Swets, 1963; Tanner, Swets, \& Green, cited in Swets, 1963; Veniar, 1958a, 1958b; Peterson \& Birdsall, Note 1). The loss so engendered will be referred to here as the uncertain-frequency (UF) effect. In the 1950s, two models were put forth to explain the results of uncertainty, these being the single-band model of Tanner (as described in Swets, 1963) ${ }^{1}$ and the multiband model described by Green (1958). The first of these assumes that the initial stage of auditory processing is a narrow-band filter with an adjustable center frequency. The multiband model assumes that all critical bands needed to cover the stimulus range are monitored continuously and that the outputs of these bands are simply added together before processing. The major datum contrary to both of these models was provided by Green in 1961. Testing with a large number of potential signals, he showed that the UF effect is considerably smaller in magnitude than predicted by either model, being no greater than $3 \mathrm{~dB}$ with 51 possible signals.

This research was first reported in a PhD dissertation submitted by the first author to the University of California, Berkeley. The authors would like to thank P. Divenyi, J. Jensen, W. Jesteadt, $W$. Kelly, and C. Watson for reading earlier versions of this paper. Grateful thanks are extended to C. Dugan and R. Yee for their help with this manuscript. The following NIH grants were beneficial to the completion of this research: 5R01NS14637. 02CMS-CD, 5R01NS14709-02CMS-CD, 5S08RR09003. Requests for reprints may be sent to the first author at the Human Communication Laboratories, Boys Town Institute, 555 North 30th Street, Omaha, Nebraska 68131.
Green (1961) proposed a third model to account for the UF effect. Here, as in the multiband model, $M$ independent bandpass filters are used by the subject to monitor, in parallel, the range of possible target frequencies. In this model, however, likelihood ratios are computed separately from the output of each filter and then combined to produce the single statistic upon which detectability depends. At any given signal level, the factor that controls UF detection is the number of bands so monitored, $\mathbf{M}$. Performance decreases nonlinearly as a function of $M$, with the increase in necessary signal energy being roughly proportional to the logarithm of the number of possibilities. ${ }^{2}$ If $\mathrm{M}$ is set by the number of "critical bands" (Fletcher, 1940; Scharf, 1970) in the space, then this model also suffers from a prediction of too great a loss with extensive uncertainty. Green's solution to this dilemma was to assert that all conditions have a degree of uncertainty so that $\mathbf{M}$ is greater than 1 for even the best of circumstances with only a single possible frequency. In this way, one can attribute the minimized effects of further uncertainty to the shallow slope of the $\log M$ function at higher values of $M$. Until recently, this has been the prevalent view of the effects of frequency uncertainty, and it is central to theoretical speculation concerning the role of uncertainty in the detection and recognition of other simple stimuli (Green \& Birdsall, 1978; Swets \& Birdsall, 1978; Swets, Green, Getty, \& Swets, 1978).

A traditional method for reducing stimulus uncertainty has been to introduce cues that, in some way, give information about the nature of the signals to be detected. Thus, lights or tones have been presented before or after observation intervals in order to tell the subject which target frequency might be or might have been presented. ${ }^{3}$ With such an approach, Greenberg (Note 2) and Swets and Sewall (1961) 
found a slight improvement in performance produced by cues that preceded the stimulus intervals (precues) but no effect from cues that came after (postcues). Greenberg argued that the relative ineffectiveness of cues in his experiment may have stemmed from the fact that the cues were themselves presented at threshold levels. In this regard, Swets and Sewall, using suprathreshold precues, found effects of $5 \%$ to $8 \%$. They found no effect from postcues. Recent work from several laboratories has supported the efficacy of cues in reducing uncertainty. Gilliom and Mills (1976) showed that auditory precues in an otherwise UF condition produced performance that approached that with a single frequency. Gilliom, Taylor, and Cline (1977) then demonstrated that auditory postcues, while less effective, also reduced the impact of UF. Similarly, using a "probecomparison" task in which a subject must detect whether a comparison tone is of the same amplitude as one of two standard tones, Kinchla (1973) found that performance with visual precues was superior to that with visual postcues. Hafter and Kaplan (Note 3) found an effect of both auditory and visual precues. In addition, they noted an interaction between motivation and uncertainty. Manipulations intended to produce a high level of motivation had a marked positive effect upon performance during UF detection but virtually no effect upon performance during cued detection.

This last finding is important to our theoretical understanding of the effects of signal uncertainty. It suggests that another factor, in addition to the potential number of signals, must influence UF detection. Hafter and Kaplan (Note 3) proposed a model that incorporates both the nonlinear effects of the factor $M$ and those of a linear operator $W$, which scales the effective bandwidths of the $M$ filters. As with Green (1961), a sensory dimension is monitored by being divided into $M$ perceptive fields, in each of which is computed a likelihood ratio. What is different is the assumption that the losses of accuracy, introduced either by pooling of bands or by misplacement of the center frequencies, are not fixed. Rather, observers may vary the effective bandwidths of the $M$ filters in order to meet the demands of the task. In one sense, a variable bandwidth has much in common with an adjustable sensory attenuator (Treisman, 1964, 1969). It is different, though, in that bandwidths are the units into which a sensory domain is divided. As above, constant detectability under conditions of UF requires that the signal be increased with the $\log$ of $\mathrm{M}$. However, since the noise increases linearly with $\mathrm{W}$, any widening of the effective bands requires an equal increase in signal strength. Thus, best performance always calls for more narrow filters rather than fewer wide ones. The concept of a variable width filter has been advanced in several previous studies (Divenyi \& Danner, 1977; Elliott,
1967; Green, 1960; Pastore \& Sorkin, 1971; Sorkin, Pastore, \& Gilliom, 1968; Swets, 1963; Swets, Green, \& Tanner, 1962). We attempt to extend this notion to the theory of UF detection.

A further assumption of the model is that the difficulty of mental effort, as in Kahneman (1973), is related to $M$, the number of independent likelihoods that must be computed. It sees the role of attention as controlling the tradeoff between the two factors $M$ and $\mathrm{W}$, with the poorer processing associated with wider bandwidths sometimes being worth the reduction in the number of bands that must be examined. Accordingly, listeners may choose various strategies for dealing with uncertainty. For example, under conditions of extreme motivation, listeners may narrow their effective bandwidths, leading to more things to "look for" but greater accuracy.

It follows directly from the theory above that any manipulation that improves the accuracy of monitoring must improve performance. However, to test the notion that this is one of the effects of uncertainty, one must vary $\mathrm{W}$ independently of $\mathrm{M}$. The purpose of the present study is to do just that-to give observers information that might improve their accuracy without changing the number of potential signal frequencies. This is to be accomplished by cues that remind listeners of the frequencies to be monitored, without telling them which frequency might be presented on any given trial. A no-cue condition is also presented here for comparison.

Single-band models that propose serial processing through an adjustable filter have had some success in accounting for the UF effect (cf. Swets, Shipley, McKey, \& Green, 1959). But other considerations have led theorists to postulate processing of more than one channel at a time. Thus, Gilliom and his colleagues (Gilliom, 1972; Gilliom \& Sorkin, 1974), studying simultaneous two-channel detection, and Shiffrin and his colleagues (cf. Shiffrin, Pisoni, \& Castaneda-Mendez, 1974), studying multichannel recognition, have devised a method that allows comparison between serial and parallel models. In this paradigm, a condition of simultaneous observation, in which any of the possible signals may occur within the observation interval, is compared with one of sequential observation, in which each type of signal may occur only within its own prescribed interval. Performance in these two conditions has proven to be identical, supporting the notion that parallel processing of simple sensory stimuli is possible. We decided to pursue this line of reasoning, testing for the effects of uncertainty under conditions of simultaneous and sequential listening. Not only would it extend the findings of Gilliom and of Shiffrin to the case of UF detection, but also the sequential paradigm would allow us to test for the possible differentiation of uncertainty into two factors, band number and bandwidth. 


\section{EXPERIMENT 1}

The first experiment was designed to resolve two questions about the uncertain-frequency effect. One concerned the usefulness of auditory precues as a means of reducing the impact of uncertainty. The second was meant as a direct test of the accuracy hypothesis, that is, the notion that a portion of the decrement normally caused by uncertainty may be attributable to a widening of the effective bandwidths. Toward this latter goal, detection was measured using special cues, described below, that were designed to reduce $\mathrm{W}$ while not changing $\mathrm{M}$. An uncued condition was also tested. Here, either of the two frequencies could appear in a single observation interval. Its purpose was to allow extension of Shiffrin's basic result to the case of UF detection.

\section{Method}

The task was to detect tone bursts presented in wide-band continuous noise, using a yes-no paradigm. The listeners were paid university students. Each was trained to asymptotic performance on every condition of an experiment before data collection began. ${ }^{4}$ Daily 2 -h sessions included eight runs of 100 experimental trials plus an indeterminate number of practice trials controlled by the subject. Only one condition was run in each session. Conditions were assigned to sessions in a counterbalanced fashion, with each observer receiving a different order.

A PDP-11/20 computer controlled the sessions. Signals were tone bursts of $50-\mathrm{msec}$ duration, with rise and fall times of $2.5 \mathrm{msec}$. The sinusoids were generated by a Wavetek 116 voltagecontrolled function generator, with the frequency set by computer to be either 500 or $1,200 \mathrm{~Hz}$. Signals were gated by a GrasonStadler 1287B electronic switch, then mixed with noise and sent to both ears via Telephonix TDH-39 headphones. In all conditions in which there were cues, the levels of the cues were $4 \mathrm{~dB}$ higher than those of the signals.

Noise was produced by a Grason-Stadler $455 \mathrm{C}$ noise generator and shaped by a Krohn-Hite $3500 \mathrm{R}$ filter set to a bandwidth of $100-1,600 \mathrm{~Hz}$. The band level of the masker was $62 \mathrm{~dB}$ SPL, producing a spectrum level (No) of $30 \mathrm{~dB}$. The noise was gated by a second Grason-Stadler 1287B electronic switch. Signals and noise were presented diotically.

Signal-to-noise ratios are defined here by the ratio of the signal's energy (E) to noise power per unit bandwidth (No). In the description of results, only the $\mathrm{E} / \mathrm{No}$ values at $500 \mathrm{~Hz}$ will be given. In order to keep detectability constant, the higher frequencies were incremented by $1 \mathrm{~dB}$ as done by Green, McKey, and Licklider (1959). Data were collected at two values of $\mathrm{E} /$ No per condition. The intent was to obtain values of $\mathrm{d}^{\prime}$ in the range $.80-1.0$ and in the range 1.2-1.50. The two points were then used independently

Table 1

Slopes (k) for Conditions and Subjects

\begin{tabular}{ccccc}
\hline & \multicolumn{3}{c}{ Certain Detection } & \\
\cline { 2 - 5 } Subject & $\begin{array}{c}\text { Single } \\
\text { Frequency* }\end{array}$ & $\begin{array}{l}\text { Constant } \\
\text { Precues } \dagger\end{array}$ & $\begin{array}{c}\text { Alternate } \\
\text { Precues }\end{array}$ & $\begin{array}{c}\text { Uncertain } \\
\text { Detection }\end{array}$ \\
\hline D.J. & 1.30 & 1.12 & 1.22 & 1.61 \\
M.H. & .99 & 1.19 & 1.31 & 1.45 \\
M.K. & 1.27 & 1.31 & 1.13 & 1.50 \\
Mean & 1.19 & 1.21 & 1.22 & 1.52 \\
\hline
\end{tabular}

Note-The uncertain detection condition was a sequential twofrequency condition with no cues. $\quad{ }^{*}$ No cues. TSequential.

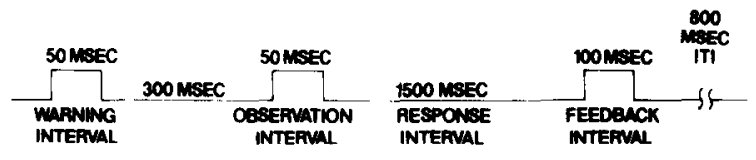

Figure 1. Trial structure for the simultaneous, two-frequency observation conditions. Warning, observation, and feedback intervals were marked by light-emitting diodes.

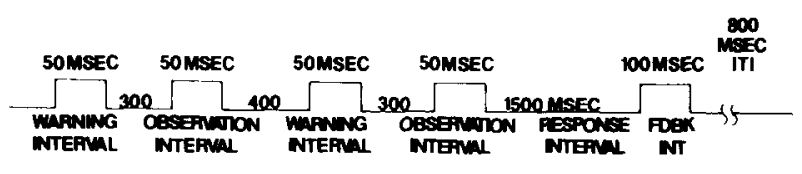

Figure 2. Trial structure for the sequential, two-frequency observation conditions. During the cued version of this condition, a $500-\mathrm{Hz}$ tone was presented during Warning Interval 1 and a 1,200-Hz tone during Warning Interval 2. Warning, observation, and feedback intervals were marked by light-emitting diodes.

to estimate a masked threshold, i.e., the $10 \log (\mathrm{E} / \mathrm{No})$ needed for a $\mathrm{d}^{\prime}=1.0$, using the relationship $\mathrm{d}^{\prime}=\mathrm{m}(\mathrm{E} / \mathrm{No})^{\mathrm{k}}$ developed by Egan, Lindner, and McFadden (1969). ${ }^{5}$ The values of the slope (k) were estimated in a separate experiment, designed only for calibration. There, psychometric functions covering a range of $4 \mathrm{~dB}$ were plotted for each subject and several representative conditions. The slopes so obtained are shown in Table 1. Those found under conditions of UF detection were greater than those determined for conditions of certainty. This fact is in accordance with theoretical slopes provided by Green and Swets (1966) and agrees with psychophysical studies of uncertainty in vision (Cohn \& Lasley, 1974).

In all conditions, a signal was a tone of one of two frequencies which might or might not occur on a given trial. In the simultaneous, two-frequency condition, the signals presented during the single observation interval were either $500 \mathrm{~Hz}$ or $1,200 \mathrm{~Hz}$. No cues were employed for this condition. The trial structure for this condition is presented in Figure 1. In the sequential, two-frequency condition, trials consisted of two observation intervals, as shown in Figure 2. If a signal occurred in the first, it was $500 \mathrm{~Hz}$; if it occurred in the second, it was $1,200 \mathrm{~Hz}$. Note the difference between the simultaneous and the sequential conditions. In the former, both frequency bands must be monitored simultaneously for best performance. In the latter, the two bands may be monitored in sequence. The psychophysical method employed in both cases was yes-no. In either case, if a signal was presented, the correct response was "yes." If not, the listeners were to respond "no." Half of the trials contained signals, with these divided equally between the two possible frequencies.

For the cued-sequential condition, a unique method of cuing was introduced. On every trial, a $500-\mathrm{Hz}$ cue was presented before the first observation interval and a $1,200-\mathrm{Hz}$ cue was presented before the second observation interval. In this way, the cues might serve to reduce the effects of inaccurate placement or widening of listening bands without revealing the identity of possible signals. Thus, $\mathbf{M}$, defined by the number of separate bands that must be monitored, would remain at two.

\section{Results}

The results of Experiment 1 are presented in Table 2. Each datum is based upon 2,000 observations. The thresholds shown are averages computed across listeners. Two planned comparisons were made using the F statistic for repeated measures and the overall within-subjects variance for an error term (Keppel, 1973). The difference of $.2 \mathrm{~dB}$ between the simultaneous and the sequential observation conditions 
Table 2

Threshold Values of E/No (in Decibels) for Experiment 1

\begin{tabular}{cccc}
\hline Subject & Condition 1 & Condition 2 & Condition 3 \\
\hline M.K. & 10.39 & 10.05 & 8.13 \\
S.A. & 8.62 & 8.08 & 7.10 \\
D.J. & 9.13 & 9.28 & 7.96 \\
Mean & 9.38 & 9.14 & 7.73 \\
\hline
\end{tabular}

Note-Condition 1 was a simultaneous two-frequency condition. Condition 2 was a sequential two-frequency condition. Condi. tion 3 was a sequential two-frequency cued condition.

(number 1 vs. number 2 ) was not significant $[F(1,4)$ $=.82, \mathrm{p}>.05]$. The cuing effect, defined by the difference between the uncued-sequential condition (number 2) and the cued-sequential condition (number 3) was $1.4 \mathrm{~dB}$. This was significant $[F(1,4)=27.0$, $\mathrm{p}<.05]^{6}$

\section{Discussion}

Note first the equality of performance produced by the simultaneous and sequential conditions of observation. This is essentially the result shown originally by Gilliom and by Shiffrin, now replicated within the UF paradigm. It says that listeners are able to monitor two frequency channels at the same time as well as in sequence. Its importance is that it rejects the single-band model of UF detection in favor of some form of parallel processing, at least in the case of two frequencies.

The most interesting result is that the auditory precues increased detection in the sequential task. Using traditional cuing methods, other investigators have reported positive effects of precues upon detection (Gilliom \& Mills, 1976; Swets \& Sewall, 1961; Greenberg, Note 2; Hafter \& Kaplan, Note 3). The improvement here, however, was obtained without reducing the number of potential signal frequencies. This supports the two-factor model of Hafter and Kaplan, since the information available in the cues could only have improved the accuracy of monitoring. The implication is that cues decreased the effective bandwidths of the maskers, thus lowering the amount of noise input to the decision process.'

\section{EXPERIMENT 2}

The second experiment was designed to investigate further the accuracy hypothesis. Suppose that only one of the two possible frequencies was cued on each trial, regardless of the frequency of the potential signal. A cue that matched the signal would be expected to improve performance, presumably by decreasing the effective bandwidth. What about a cue that did not match the potential signal? Would the reduction of bandwidth at the cued, but incorrect, frequency affect processing of the uncued, but correct, frequency?
To answer this question, a different paradigm was used. Here, cues alternated in frequency between trials in a predictable fashion, with all odd-numbered trials cued by a $500-\mathrm{Hz}$ tone and all even-numbered trials cued by a $1,200-\mathrm{Hz}$ tone. Thus, on $25 \%$ of the trials the cue matched the signal (a "good" cue), on $25 \%$ it did not (a "bad" cue), and on $50 \%$ it preceded an interval with no signal. Subjects answered only whether or not a signal was present, regardless of frequency.

\section{Method}

The method of this experiment was the same as that of Experiment 1 , except where noted. There were four experimental conditions, two uncued and two cued. Numbers 1 and 2 were the simultaneous, two-frequency condition and the sequential, twofrequency condition, respectively, of Experiment 1 . Conditions 3 and 4 were the cued counterparts of 1 and 2, respectively. The cuing frequency alternated with trials. Thus, in the sequential case, the cue alternated in position, being before either Observation Interval 1 or Observation Interval 2, but not both.

The data from the cued conditions were compiled separately according to whether the cue was a match or a mismatch with signal. This produced six categories: Condition 1, Condition 2, Condition 3M (match), Condition 3MM (mismatch), Condition $4 \mathrm{M}$ (match), and Condition $4 \mathrm{MM}$ (mismatch). The estimates of threshold from Conditions 1 and 2 were based upon 2,400 observations ( 24 runs of 100 trials), while those with cues were based upon 1,200 observations $(24 \times 50) .^{8}$

\section{Results}

The results are shown in Table 3. As before, the small difference of $.2 \mathrm{~dB}$ between Condition 1 (simultaneous, two-frequency) and Condition 2 (sequential, two-frequency) was not statistically significant $[F(1,10)=.26, p>.05]$. The difference between Condition 1 and Condition $3 \mathrm{M}$, the cue-match effect, was $1.5 \mathrm{~dB}$ and significant $[\mathrm{F}(1,10)=9.24$, $\mathrm{p}<.05$ ]. The difference between Condition 1 and Condition 3MM, the cue-mismatch effect, was $-.9 \mathrm{~dB}$, which was marginally significant $[\mathrm{F}(1,10)$ $=3.32, .05<p<.101$. Condition 2 differed from Condition $4 \mathrm{M}$ by $1.3 \mathrm{~dB}$, which was significant $[F(1,10)=7.13, p<.05]$. Conditions 2 and $4 \mathrm{MM}$ differed by $-1.1 \mathrm{~dB}$, which was also marginally significant $[F(1,10)=4.42, .05<\mathrm{p}<.10]$.

Table 3

Threshold Values of E/No (in Decibels) for Experiment 2

\begin{tabular}{lcccccc}
\hline & \multicolumn{6}{c}{ Condition } \\
\cline { 2 - 7 } jub- & 1 & 2 & $3 \mathrm{M}$ & 3MM & 4M & 4MM \\
\hline M.K. & 9.24 & 9.43 & 7.75 & 9.40 & 8.84 & 10.07 \\
D.J. & 9.40 & 9.31 & 7.91 & 10.75 & 8.38 & 10.10 \\
M.H. & 8.89 & 9.56 & 7.28 & 10.13 & 7.05 & 11.31 \\
Mean & 9.18 & 9.43 & 7.65 & 10.09 & 8.09 & 10.49 \\
\hline
\end{tabular}

Note-Condition 1 was a simultaneous two-frequency condition. Condition 2 was a sequential two-frequency condition. Condition $3 M$ was a simultaneous two-frequency cue-match condition. Condition 3MM was a simultaneous two-frequency cue-mismatch condition. Condition $4 M$ was a sequential two-frequency cuematch condition. Condition $4 M M$ was a sequential two-frequency cue-mismatch condition. 


\section{Discussion}

Because simultaneous and sequential performance under uncertainty do not differ, a discussion of the effects of cuing need not make that distinction. What we see is that, for both cases, good cues improved detection, while bad cues harmed it. The improvements of about $1.5 \mathrm{~dB}$ were of approximately the same magnitude as in Experiment 1, suggesting once more that cues can narrow the effective listening bands. When the cue did not match the signal, performance deteriorated by approximately $1 \mathrm{~dB}$. This occurred despite the fact that all listeners were well trained and knew that the signal on each trial could be either the cued frequency or the other frequency. It seems, then, that while the cue sharpened the listening band at one frequency region, it somehow impaired processing at the other.

This negative effect may appear to result from a serial process. Perhaps, for example, the effect of a cue is to cause the perceiver to act as a single-band detector set to the cued frequency. In this case, the detector would be off-target when the cue was bad and, thus, performance would be impaired. It is highly unlikely, however, that this interpretation is valid. First, this hypothesis would predict that the negative effect of bad cues would be reduced or nonexistent for the sequential condition, in which the two frequency regions are monitored serially. In fact, both conditions showed the negative effect, with the magnitude of the effect greater for the sequential condition. Second, a control experiment was performed explicitly to test this single-band hypothesis, with negative results. ${ }^{9}$ Thus, while we feel we understand the increments with the matched cues, we have no ready explanation for why there should have been a decrement with the others.

\section{SUMMARY}

Detection of one of two possible auditory frequencies was shown to be the same whether the conditions of presentation were simultaneous, with either frequency possible in a single observation interval, or sequential, when one possible frequency could be presented only in the first of two intervals and the other possible frequency only in the second. This demonstration, which has been shown by others for various kinds of stimuli, is taken as evidence of the ability to attend to two frequency bands simultaneously.

The nature of uncertainty was studied by applying various types of auditory cues to the two-frequency problem. These were designed to test a model that breaks the effects of uncertainty into two factors. One is $\mathbf{M}$, the number of different signals that might possibly be presented. Performance decreases with the log of the $\mathrm{M}$ bands that must be monitored. The other factor is $\mathrm{W}$, the bandwidths of the $\mathrm{M}$ per- ceptual bands. W limits the accuracy of performance in each band by determining the amount of noise. It is thought to be affected by cues that improve the accuracy of search. The cues used here were special, being designed to reduce $\mathrm{W}$ without changing $\mathrm{M}$. The results confirmed that performance could be improved by sharpening the listening bands without removing the fundamental uncertainty.

In a second experiment, it was shown that when only one possible frequency was cued on a given trial, detection of the other dropped, even below the control condition of no cue at all. This somewhat curious result may suggest that when cues are used to improve accuracy, subjects may actually widen the bands at other possible signal sites.

\section{REFERENCE NOTES}

1. Peterson, W. W., \& Birdsall, T. G. The theory of signal detectability. Unpublished report, Engineering Research Institute, University of Michigan, Ann Arbor, Michigan, 1953.

2. Greenberg, G. Z. Cueing signals and frequency uncertainty in auditory detection. Unpublished report, Hearing and Communication Laboratory, Indiana University, Bloomington, Indiana, 1962.

3. Hafter, E. R., \& Kaplan, R. A. The interaction between motivation and uncertainty as a factor in detection. NASA project report, Ames Research Center, Moffit Field, California, 1976.

\section{REFERENCES}

Cohn, T. E., \& LAsley, D. J. Detectability of a luminance increment: Effects of spatial uncertainty. Journal of the Optical Society of America, 1974, 64, 1715-1719.

Divenyi, P. L., \& Danner, W. F. Discrimination of time intervals marked by brief acoustic pulses of various intensities and spectra. Perception \& Psychophysics, 1977, 21, 125-142.

Egan, J. P., Lindner, W. A., \& McFadden, D. Maskinglevel differences and the form of the psychometric function. Perception \& Psychophysics, 1969, 4, 209-215.

ELLIoTT, L. L. Development of auditory narrow-band frequency contours. Journal of the Acoustical Society of America, 1967, 42, 143-153.

Fletcher, H. Auditory patterns. Review of Modern Physics, $1940,12,47-65$

GILI rom, J. D. Binaural detection in a sequential detection paradigm. Journat of the Acoustical Society of America, 1972, 52, 167(A).

Giliıom, J. D., \& Milıs, W. M. Information extraction from contralateral cues in the detection of signals of uncertain frequency. Journal of the Acoustical Society of America, 1976. $59,1428-1433$.

Giliım, J. D., \& Sorkin, R. D. Sequential vs. simultaneous two-channel signal detection: More evidence for a high-level interrupt theory. Journal of the Acoustical Society of America, 1974, 56, 157-164.

Gilıom, J. D. TAYlor, D. W., \& Cline, C. Should an informational cue precede or follow the signal event: Results with a contralateral cue in an uncertain frequency paradigm. Journal of the Acoustical Society of America, 1977, 61, S62(A).

GREFN, D. M. De1ection of multiple component signals in noise. Journal of the Acoustical Society of America, 1958, 30, 904-911.

Grasen, D. M. Auditory detection of a noise signal. Journal of the Acoustical Society of America, 1960, 32, 121-131.

GRFFN, D. M. Detection of auditory sinusoids of uncertain fre- 
quency. Journal of the Acoustical Society of America, 1961, 33, 897-903.

Green, D. M., \& Birdsall, T. G. Detection and recognition. Psychological Review, 1978, 85, 192-200.

Green, D. M., McKey, M. H., \& Lickliner, J. C. R. Detection of a pulsed sinusoid in noise as a function of frequency. Journal of the Acoustical Society of America, 1959, 31, 14461452.

Green, D. M., \& Swets, J. A. Signal detection theory and psychophysics. Huntington, N.Y: Krieger, 1966.

Kahneman, D. Attention and effort. Englewood Cliffs, N.J: Prentice-Hall, 1973.

KePpez, G. Design and analysis: A researcher's handbook. Englewood Cliffs, N.J: Prentice-Hall, 1973.

KinCHLA, R. A. Selective processes in sensory memory: A probecomparison procedure. In S. Kornblum (Ed.), Attention and performance IV. New York: Academic Press, 1973.

Nolte, L. W., \& JaARsma, D. More on the detection of one of M orthogonal signals. Journal of the Acoustical Society of America, 1967, 41, 497-505.

Pastore, R. E., \& Sorkin, R. D. Adaptive auditory signal processing. Psychonomic Science, 1971, 23, 259-260.

RUNYon, R. P., \& HABER, A. Fundamentals of behavioral statistics. Menlo Park, Calif: Addison-Wesley, 1977.

Scharf, B. Critical bands. In J. R. Tobias (Ed.), Foundations of modern auditory theory (Vol. 1). New York: Academic Press, 1970.

Shiffrin, R. M., Pisoni, D. B., \& Castaneda-Mendez, K. Is attention shared between the ears? Cognitive Psychology, $1974,6,190-215$.

Sorkin, R. D., Pastore, R. E., \& Gilliom, J. D. Signal probability and the listening band. Perception \& Psychophysics, 1968, 4, 10-12.

Swets, J. A. Central factors in auditory-frequency selectivity. Psychological Bulletin, 1963, 60, 429-440

Swets, J. A., \& Birdsall, T. G. Repeated observation of an uncertain signal. Perception \& Psychophysics, 1978, 23, 269-274.

Swets, J. A., Green, D. M., GetTy, D. J., \& Swets, J. B. Signal detection and identification at successive stages of observation. Perception \& Psychophysics, 1978, 23, 275-289.

Swets, J. A., Green, D. M., \& Tanner, W. P., Jr. On the width of critical bands. Journal of the Acoustical Society of America, 1962, 34, 108-113.

Swets, J. A., \& Sewall, S. T. Stimulus vs. response uncertainty in recognition. Journal of the Acoustical Society of America, 1961, 33, 1586-1592.

Swets, J. A., Shipley, E. F., McKey, M. J., \& Green, D. M. Multiple observations of signals in noise. Journal of the Acoustical Society of America, 1959, 31, 514-521.

TAYLOR, M. M. Detectability theory and the interpretation of vigilance data. Acta Psychologica, 1967, 27, 390-399.

TAYloR, M. M., \& Clarke, D. P. J. Monaural detection with contralateral cue (MDCC). Il. Interaural delay of cue and signal. Journal of the Acoustical Society of America, 1971, 49, 1243-1253.

TAYLoR, M. M., \& Forees, S. M. Monaural detection with contralateral cue (MDCC). I. Better than energy detector performance by human observers. Journal of the Acoustical Society of America, 1969, 46, 1519-1526.

Taylor, M. M., Clarke, D. P. J., \& Smith, S. M. Monaural detection with contralateral cue (MDCC). III. Sinusoidal signals at a constant performance level. Journal of the Acoustical Society of America, 1971, 49, 1795-1804.

Taylor, M. M., Smith, S. M., \& Clarke, D. P. J. Monaural detection with contralateral cue (MDCC). IV. Psychometric functions with sinusoidal signals. Journal of the Acoustical Society of America, 1971, 50, 1151-1161.

Treisman, A. M. Selective attention in man. British Medical Bulletin, 1964, 20, 12-16.

Treisman, A. M. Strategies and models of selective attention. Psychological Review, 1969, 76, 282-299.
Veniar, F. A. Signal detection as a function of frequency ensemble. I. Journal of the Acoustical Society of America, 1958, 30, 1020-1024. (a)

Veniar, F. A. Signal detection as a function of frequency ensemble. II. Journal of the Acoustical Society of America, 1958, 30, 1075-1078. (b)

\section{NOTES}

1. The original work by Tanner, Swets, and Green (1956) went unpublished. The Swets (1963) paper presents both the theory and the summary data.

2. This was derived for the ideal detector by Peterson and Birdsall (Note 1), who showed that the relationship between signal energy and the number of potential signals, for a constant $d^{\prime}$, is:

$$
2 E / N o=\ln \left\{1+M\left[\exp \left(d^{\prime 2}\right)-1\right]\right\}
$$

where No is the spectral level of the noise. This derivation is an admitted first approximation. For extreme levels of uncertainty or $d^{\prime}$, the derivation by Nolte and Jaarsma (1967) provides a better fit.

3. Taylor and his colleagues have employed simultaneous, contralateral cues to improve detection in a nonuncertainty paradigm. They have studied both noise signals (Taylor \& Clarke, 1971; Taylor \& Forbes, 1969) and tones (Taylor, Clarke, \& Smith, 1971; Taylor, Smith, \& Clarke, 1971). In some conditions, they have noted performance that approached that of an ideal detector with signal known exactly. The relationship between these results and other binaural phenomena is discussed by the authors. Because of the obvious binaural interaction present and because the research paradigm was not one of uncertainty, we shall not discuss their work further in this paper.

4. Data in support of this assertion will be provided by the first author upon request.

5. We are aware that the interpretation of $d^{\prime}$ under conditions of stimulus uncertainty is sometimes problematical (Taylor, 1967). In this specific instance, however, such considerations do not apply.

6. The results discussed above have been replicated. In later experiments, a single-frequency condition $(M=1)$ was also run. There were no significant differences between cued conditions and single-frequency conditions.

7. It has been suggested that these results may be interpretable by a model that considers the cue as enhancing an internal representation of the signal. Although we cannot think of an unambiguous test of this interpretation, we do not discount its possible validity

8. The designation of a condition as either $\mathbf{M}$ or $\mathbf{M M}$ was contingent upon signal events alone. The false-alarm rates used in the calculation of $d^{\prime}$ were computed from noise-only trials and were, therefore, the same for both $M$ and MM conditions. Response criterion was, by default, assumed to be constant across both $\mathrm{M}$ and $\mathrm{MM}$ conditions.

9. A control experiment was performed to test the hypothesis that cues cause the listener to adopt, albeit involuntarily, a singleband strategy. If this were true, a listener would be expected to perform more poorly when multiple frequency regions were cued simultaneously than when they were cued in sequence.

Two conditions were run. As before, the task was to detect tones of one of two possible frequencies. Condition 1 was identical to the simultaneous, two-frequency condition described above, with one exception. Both signal frequencies were cued simultaneously during the warning interval. That is, a two-frequency "chord" was presented before the observation interval as a cue. Number 2 was identical to the cued version of the sequential, two-frequency condition presented in Experiment 1. Two new listeners were used. All other methodological details were the same as those described above. 
Table 4

Threshold Values of E/No (in Decibels) for Experiment 3

\begin{tabular}{ccc}
\hline Subject & Condition $1^{*}$ & Condition $2 \dagger$ \\
\hline L.H. & 8.08 & 8.16 \\
D.J. & 7.42 & 7.14 \\
K.B. & 9.37 & 9.36 \\
Mean & 8.29 & 8.22 \\
\hline
\end{tabular}

*Simultaneous cues. $\quad$ tSequential cues.
The results are shown in Table 4 . Each datum represents 1,200 observations. A t test for correlated samples was performed (Runyon \& Haber, 1977). The slight difference in performance between the two conditions was not statistically reliable $[t(2)$ $=.65, \mathrm{p}>.05]$. There was, therefore, no evidence to support the hypothesis described above.

(Received for publication September 17, 1979; revision accepted April 28, 1980.) 